\title{
Is motor imagery effective for gait rehabilitation after stroke? A Cochrane Review summary with commentary
}

\author{
Ekin Ilke Sen \\ Department of Physical Medicine and Rehabilitation, Istanbul Faculty of Medicine, Istanbul University, \\ Capa, Istanbul, Turkey \\ E-mail: ekinozgorgu@gmail.com
}

\begin{abstract}
.
BACKGROUND: Motor imagery (MI) is a promising therapeutic technique for stroke rehabilitation.

OBJECTIVE: To assess the effects of MI on gait rehabilitation after stroke.

METHODS: To summarize the "Cochrane Review" by Silva et al.

RESULTS: Twenty-one studies with 762 participants were included in the Cochrane review. Very low level of certainty evidence pointed to some beneficial effects of MI alone or combined with action observation or physical practice on walking speed compared to other therapies. There is uncertainty about the effect of MI compared to other therapies in terms of motor function or functional mobility.

CONCLUSIONS: High-quality adequately powered studies investigating the effects of MI in individuals with stroke should be encouraged.
\end{abstract}

Keywords: Motor imagery, rehabilitation, stroke, gait

The aim of this commentary is to discuss from a rehabilitation perspective the Cochrane Review "Motor imagery for gait rehabilitation after stroke" (Silva et al., 2020) by Silva, Borges, Santiago, Lucena, Lindquist, \& Ribeiro, ${ }^{\text {a }}$ which was recently published by the Cochrane Stroke Group. This

\footnotetext{
${ }^{a}$ This summary is based on a Cochrane Review previously published in the Cochrane Database of Systematic Reviews 2020, Issue 9, Art. No.:CD013019, DOI: 10.1002/14651858.CD013019.pub2 (see www.cochranelibrary.com for information). Cochrane Reviews are regularly updated as new evidence emerges and in response to feedback, and Cochrane Database of Systematic Reviews should be consulted for the most recent version of the review.

The views expressed in the summary with commentary are those of the Cochrane Corner author and do not represent the Cochrane Library or Wiley.
}

Cochrane Corner is produced in agreement with $\mathrm{Neu}$ roRehabilitation by Cochrane Rehabilitation.

\section{Background}

Stroke is a major cause of disability and mortality worldwide, and its prevalence and burden will likely increase with progressive ageing. The Global Burden of Disease study revealed that stroke was the leading cause of disability-adjusted life years among the neurological disorders (GBD 2016 Neurology Collaborators, 2019). The effects of a stroke include paralysis and muscle weakness in the lower limbs as well as a reduced balance and mobility. As a result, 
stroke survivors may have persistent difficulties in performing daily living activities and participating in social and community activities.

Independent, balanced gait is one of the main goals of all rehabilitation programs for individuals with reduced balance and mobility after stroke. Rehabilitation approaches in which motor control of the gait cycle is relearned by activation of the motor network are important for functional recovery. Motor imagery (MI), in which individuals engage in a cognitively rehearsed task in a safe, repetitive manner without actual execution of the movement, is a promising therapeutic technique. MI has been shown to be effective for the functional rehabilitation of lower limbs as well as the recovery of daily activities when applied in combination with conventional physical therapy (García Carrasco \& Aboitiz Cantalapiedra, 2016). However, the optimal intervention protocols remain unclear. Furthermore, there is a high level of heterogeneity in the methodological quality of relevant studies and conflicting results regarding the effects of MI in the recovery of individuals after stroke (Guerra, Lucchetti, \& Lucchetti, 2017). Recently, a new Cochrane Review by Silva et al. (2020), has specifically addressed the question of whether MI can improve gait among stroke survivors.

\section{Objective}

The aim of this Cochrane Review was to explore whether MI had any treatment effects on improving ability to walk in stroke survivors.

\section{What was studied and methods}

The population addressed in this review was adults aged 18 years and over with any type of stroke at any stage with varying degrees of severity (excluding mixed etiologies such as acquired brain injury). The intervention studied was MI alone or associated with action observation, functional gait training, or physical activity. The intervention was compared to other therapies (including conventional physical therapy), placebo, or no therapy. The primary outcome studied was "walking ability" as tested by walking speed (measured by biomechanical analysis or walking tests) and dependence on personal assistance (the level of dependence having been defining using reported data from walking related functional scales). The secondary outcomes were walking endurance, motor function, functional mobility, and adverse events. The review authors searched for randomized controlled trials that had been published up to February 2020.

\section{Results}

The review included 21 studies with a total of 762 participants. Only 11 of the 21 studies had data which could be included in the meta-analyses. None of the included studies compared MI with placebo or no therapy, so the results all relate to the effect of MI compared to other therapies.

The review shows that:

- Motor imagery had a positive effect on walking speed compared with other therapies at the end of treatment (very low certainty evidence). MI was more beneficial for improving walking speed regardless of the stage of stroke, the type of treatment, and the dependence on personal assistance.

- Motor imagery had no greater effect than other therapies on motor function and functional mobility at the end of the treatment (very low certainty evidence).

- There was insufficient evidence to reach conclusions about the effect of MI on the dependence on personal assistance and walking endurance.

- Also, due to the lack of follow-up data in the included studies, it was not possible to define the medium- or long-term effects of MI on the outcome measures.

- A limited number of studies (seven trials) reported no adverse events including pain, falls, or all-cause deaths related to the interventions.

\section{Conclusions}

The authors concluded that, compared with other therapies, MI alone or associated with action observation or physical practice may increase walking speed within a short time period based on very low certainty evidence. They also found very low certainty evidence that MI was no more beneficial than other therapies in terms of motor function or functional mobility over a short time period. There was insufficient evidence to determine the effects of MI on mentioned outcomes in comparison to placebo or no intervention. MI seemed not to have any adverse 
effects. They further stated that caution is needed to interpret the conclusion as many studies had methodological limitations, small sample sizes, and wide confidence intervals.

\subsection{Implications for practice in neurorehabilitation}

Given that the certainty of evidence from the included studies in the Cochrane Review (Silva et al., 2020) was judged to be very low, it is uncertain whether MI alone or associated with action observation or physical practice improves gait after stroke. Additionally, it is unclear which treatment protocol is best, how long the treatment effect lasts, and which patient profile is optimal among stroke survivors. Therefore, high-quality trials need to be conducted to determine the treatment effects of MI for gait rehabilitation after stroke. Moreover, estimating the rehabilitation effects of MI on walking ability is complex due to the possibility of spontaneous recovery as a result of the interventions. Since the review authors did not find any studies comparing MI to a placebo or no intervention, this could be another topic for future research that could be suggested to have an appropriate design such as a crossover design for not leaving any patient without a treatment. From a rehabilitation perspective, the use of International Classification of Functioning, Disability and Health Core Sets for stroke (Geyh et al., 2004), particularly those relevant to participation and environmental factor components (Salter et al., 2005), would enable analysis of the impact of MI on specific components of disability in order to determine the needs of each patient and optimize the recovery process.

\section{Acknowledgments}

The author thanks Cochrane Rehabilitation and Cochrane Stroke Group for reviewing the contents of the Cochrane Corner.

\section{Conflict of interest}

The author declares no conflicts of interest.

\section{References}

García Carrasco, D., \& Aboitiz Cantalapiedra, J. (2016). Effectiveness of motor imagery or mental practice in functional recovery after stroke: a systematic review. Neurologia (Barcelona, Spain), 31(1), 43-52. https://doi.org/10.1016/j.nrl.2013.02. 003

GBD 2016 Neurology Collaborators (2019). Global, regional, and national burden of neurological disorders, 1990-2016: a systematic analysis for the Global Burden of Disease Study 2016. The Lancet Neurology, 18(5), 459-480. https://doi.org/ 10.1016/S1474-4422(18)30499-X

Geyh, S., Cieza, A., Schouten, J., Dickson, H., Frommelt, P., Omar, Z., Kostanjsek, N., Ring, H., \& Stucki, G. (2004). ICF Core Sets for stroke. Journal of Rehabilitation Medicine, (44 Suppl), 135-141. https://doi.org/10.1080/16501960410016776

Guerra, Z. F., Lucchetti, A., \& Lucchetti, G. (2017). Motor Imagery Training After Stroke: A Systematic Review and Meta-analysis of Randomized Controlled Trials. Journal of Neurologic Physical Therapy: JNPT, 41(4), 205-214. https://doi.org/10.1097/ NPT.0000000000000200

Salter, K., Jutai, J. W., Teasell, R., Foley, N. C., Bitensky, J., \& Bayley, M. (2005). Issues for selection of outcome measures in stroke rehabilitation: ICF Participation. Disability and Rehabilitation, 27(9), 507-528. https://doi.org/10.1080/096382804 0008552

Silva, S., Borges, L. R., Santiago, L., Lucena, L., Lindquist, A. R., \& Ribeiro, T. (2020). Motor imagery for gait rehabilitation after stroke. Cochrane Database of Systematic Reviews, 9, CD013019. https://doi.org/10.1002/14651858.CD013019. pub2 